\title{
Case Report \\ Severe Acral Necrosis Complicating Thrombotic Microangiopathy in Two Toddlers
}

\author{
H. Nassih $\left(\oplus,{ }^{1}\right.$ Z. Lazrak, ${ }^{1}$ and S. Younous ${ }^{2}$ \\ ${ }^{1}$ From B Pediatric Ward, Department of Pediatrics, Child and Mother Hospital, Mohammed VI University Hospital Center, \\ Marrakesh Medical and Pharmacy Faculty, Caddy Ayad University, Marrakesh, Morocco \\ ${ }^{2}$ From the Pediatric Intensive Care Unit, Department of Pediatrics, Child and Mother Hospital, \\ Mohammed VI University Hospital Center, Marrakesh Medical and Pharmacy Faculty, Caddy Ayad University, \\ Marrakesh, Morocco
}

Correspondence should be addressed to H. Nassih; houda.ped@gmail.com

Received 1 May 2020; Revised 16 November 2020; Accepted 5 December 2020; Published 15 December 2020

Academic Editor: Carmelo Romeo

Copyright (c) $2020 \mathrm{H}$. Nassih et al. This is an open access article distributed under the Creative Commons Attribution License, which permits unrestricted use, distribution, and reproduction in any medium, provided the original work is properly cited.

Acral ischemia/necrosis is one of the rarest but most dreadful complications of thrombotic microangiopathy in pediatric patients. It is more reported with thrombotic thrombocytopenic purpura than with hemolytic and uremic syndrome. Even with anticoagulant therapy, it is often irreversible, leading to amputation.

\section{Introduction}

Thrombotic microangiopathy (TM) leads to microcirculatory thrombosis. Ischemic changes that follow are more frequently seen in the central nervous and renal systems. Musculoskeletal system involvement is less described. However, when it occurs, acral necrosis is often severe. We report two toddlers presenting with an acute onset of TM complicated by bilateral acral ischemia.

\section{Case Report}

2.1. Case One. An eleven-month-old and first-degree consanguineous boy was admitted to the pediatric intensive care unit (PICU) for acute neurological distress. He was treated for pneumonia ten days ago and had taken oral amoxicillin $100 \mathrm{mg} / \mathrm{kg} /$ day. His antipneumococcal vaccination was up to date. The mother reported rare-black urines during the last three days. The last 24 hours before admission, the child was drowsy and hypotonic. At admission to the pediatric emergency department, first clinical evaluation found a comatose child with a Glasgow score of 8/15. He had generalized edema, pallor, and dyspnea. He had normal body temperature of $36.5^{\circ} \mathrm{C}$. His pulseoximetry found $\mathrm{SpO}_{2}$ of $75 \%$. He also had hypertension of $167 / 87 \mathrm{mmHg}$. After intubation and mechanical ventilation, chest radiograph was performed and was normal. Blood, urine, stool, and bronchial samples were collected for bacteriological tests. He was oliguric at $0.3 \mathrm{cc} / \mathrm{kg} / \mathrm{h}$. Intravenous furosemide was given at $6 \mathrm{mg} / \mathrm{kg} /$ day. Because of the onset of coma, pallor, and oliguria after an episode of upper respiratory tract infection, thrombotic thrombocytopenic purpura (TTP) was highly suspected. Blood tests found severe hemolytic anemia (hemoglobin of $4 \mathrm{~g} / \mathrm{dl}$, haptoglobin of $0.01 \mathrm{~g} / \mathrm{l}$, reticulocytes of $134000 / \mathrm{mm}^{3}$, LDH of $2390 \mathrm{UI} / \mathrm{l}$, and ferritin of $985 \mathrm{ug} / \mathrm{ml}$ ), thrombocytopenia (platelets of $79000 / \mathrm{mm}^{3}$ ), acute renal failure, hyperkalemia, and acidosis (urea of $2.04 \mathrm{~g} / \mathrm{l}$, creatinine of $19 \mathrm{mg} / \mathrm{l}, \mathrm{K}^{+}$of $7 \mathrm{mmol} / \mathrm{l}, \mathrm{HCO}^{3-}$ of $8 \mathrm{mmol} / \mathrm{l}$, and $\mathrm{pH}$ of 7.1). Meanwhile, his CRP and ESR were normal (respectively, $2 \mathrm{mg} / \mathrm{l}$ and $6 \mathrm{~mm}$ the first hour). After catheter placement, continuous peritoneal dialysis was started. The child was transfused with blood and put under nicardipine per IV. Bronchial, blood, and stool samples were sterile. On the second day of his admission to the PICU, the child was still anuric with less than $1 \mathrm{cc} / \mathrm{kg} /$ day of urine output.

Meanwhile, he developed acral ischemia of the right leg, which was rapidly extensive within a few hours (Figure 1(a)). The Doppler sonography found a thrombus of the right 


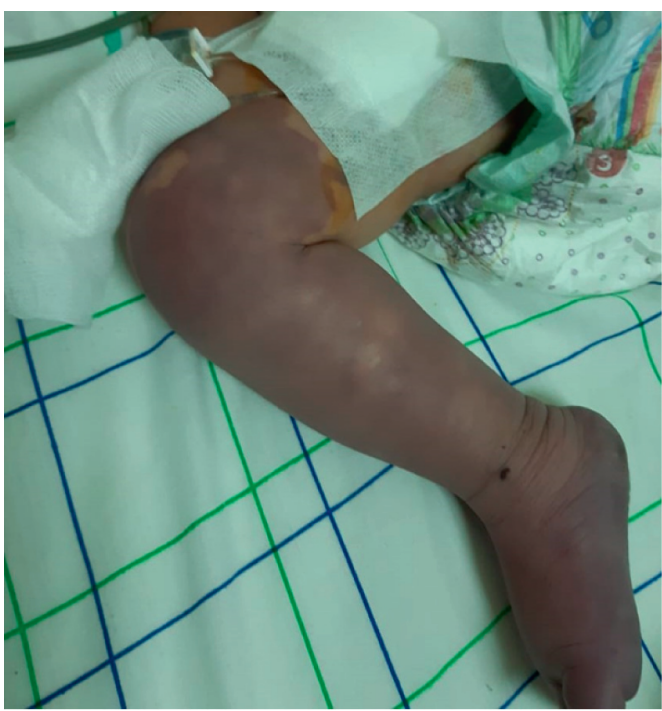

(a)

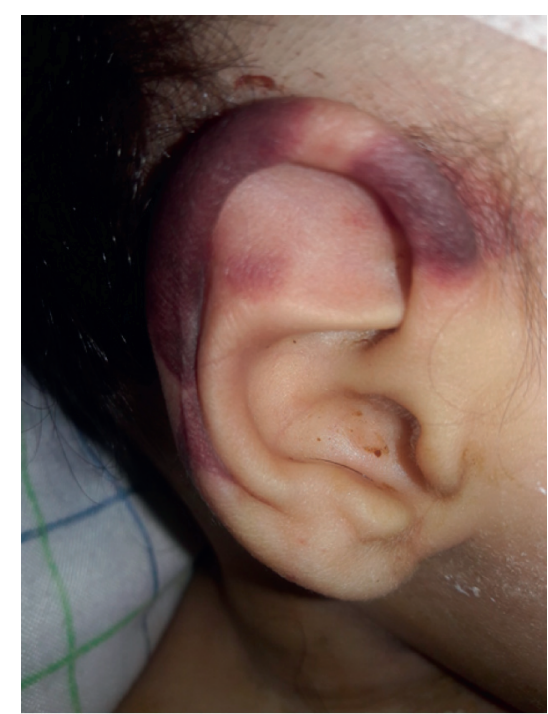

(b)

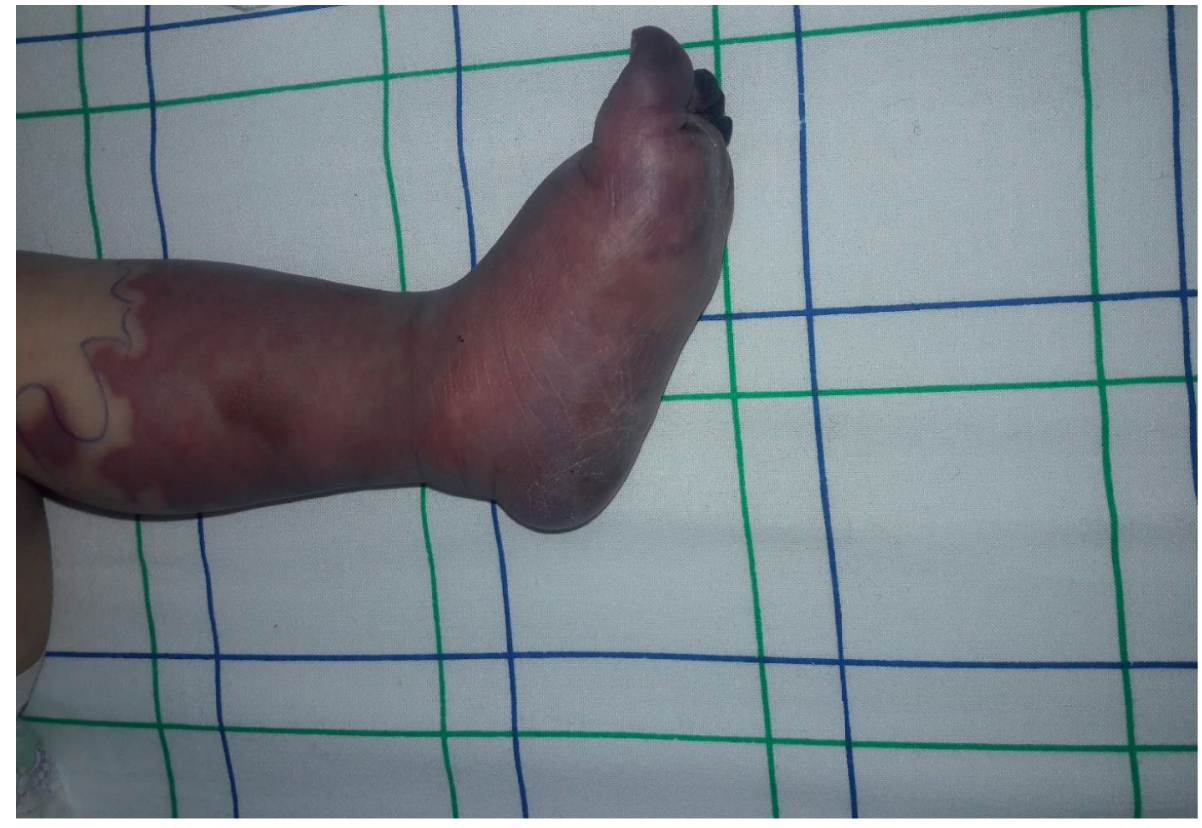

(c)

FIGURE 1: (a) An eleven-month-old boy with acral necrosis of the right foot. (b, c) A seven-month-old boy with distal necrosis of the foot and ears.

external iliac artery. Permeabilization surgery was performed, and intravenous unfractionated heparin (initially $75 \mathrm{UI} / \mathrm{kg}$, then by continuous intravenous infusion at $20 \mathrm{UI} /$ $\mathrm{kg} / \mathrm{h}$ ) was started. On the third day of hospitalization, the ischemia extended to the left leg. The thrombophilia workup was unremarkable (PTT, aPTT, INR, fibrinogen, D-Dimers, antithrombin III, and protein $\mathrm{C} / \mathrm{S}$ ), as well as the $\mathrm{C} 3 / \mathrm{C} 4$ complement fractions. Unfortunately, hemodynamic instability and neurological deterioration kept worsening. We lost the child after three days of PICU hospitalization.

2.2. Case Two. A seven-month-old boy was admitted to PICU for acute respiratory distress. Fifteen days ago, he had acute bloody diarrhea and fever of $40^{\circ} \mathrm{C}$. His doctor suspected dysentery of bacterial origin and prescribed oral antibiotics (sulfamethoxazole-trimethoprim) for five days. Afterwards, the boy regained apyrexia, and the stool was back to normal. But, by the tenth day, the child developed generalized edema, gross hematuria, and oliguria. He was admitted to the pediatric emergency department, and first evaluation found a conscious child, with a Glasgow score of $13 / 15$. He had severe respiratory distress, with hypoxia (respiratory rate of 65 cycles/min, and $\mathrm{SpO} 2$ of $51 \%$ ) and pallor. He was apyretic at $37^{\circ} \mathrm{C}$ and had hypertension of 154 / $89 \mathrm{mmHg}$. Chest radiograph found signs of acute pulmonary edema. He was anuric even after ureteral catherization and an intravenous bolus of $5 \mathrm{mg} / \mathrm{kg}$ of furosemide. The 
respiratory state kept worsening despite noninvasive ventilation, so the child was intubated. Laboratory workup revealed severe acidosis $\left(\mathrm{HCO}^{3-}\right.$ of $5 \mathrm{mmol} / \mathrm{l}$ and $\mathrm{pH}$ of 7$)$, acute renal failure (urea of $1.9 \mathrm{~g} / \mathrm{l}$, creatinine of $18.4 \mathrm{mg} / \mathrm{l}$ ), hyponatremia, and hyperkalemia $\left(\mathrm{K}^{+}\right.$of $5.6 \mathrm{mmol} / \mathrm{l}$ and $\mathrm{Na}^{+}$ of $125 \mathrm{mmol} / \mathrm{l}$ ). Normochromic-normocytic-anemia (hemoglobin of $5.5 \mathrm{~g} / \mathrm{dl}$ ) and thrombocytopenia (platelets of $36000 / \mathrm{mm}^{3}$ ) were found on complete cell blood count. Meanwhile, CRP and ESR were normal. Haptoglobin was indetectable, and reticulocyte count, $\mathrm{LDH}$, and ferritin levels were very high (respectively, $165200 / \mathrm{mm}^{3}, 1450 \mathrm{UI} / \mathrm{l}$, and $735 \mathrm{ug} / \mathrm{ml}$ ), pointing towards hemolytic and uremic syndrome (HUS). Complement fractions (C3/C4/CH50) were within normal range. Peritoneal dialysis was started, along with intravenous nicardipine. Blood and platelets transfusion were performed. Coprology and PRC of stool were negative. At the third day of PICU stay, we noticed rapidly extensive bilateral acral ischemia of the feet, hands, and ears (Figures 1(b) and 1(c)). The child was put on continuous intravenous unfractionated heparin. After twenty-four hours of starting heparin therapy, ischemia was still extensive and had reached the two knees. The thrombophilia workup was normal. After seven days of the PICU stay, the child died because of persistent hemodynamic instability despite adequate management.

\section{Discussion}

TM, which broadly includes TTP and HUS, is a multisystemic disorder characterized by thrombocytopenia, microangiopathic hemolytic anemia, and ischemic manifestations resulting from platelet agglutination in the arterial microvasculature $[1,2]$. Acral necrosis (distal necrosis of fingers and toes), also called peripheral digit ischemic syndrome (PDIS), usually occurs as a sequel to severe Raynaud's phenomenon, a vasospastic disorder frequently related to endothelial cell dysfunction [3]. It is uncommon in pediatric TM [4]; its physiopathology is also unclear [5]. One study reported that, among 94 adult patients with TM, PDIS developed in six of them, and in all these patients, PDIS occurred with postoperative TTP. There were neither arterial nor venous microthrombi observed in any of these patients [6]. The first case described was our eleven-month-old boy. We found no thrombophilia disorder in these children. The lack of facility made MA workup (ADAMST 13 activity, antifactor $\mathrm{H}$ antibodies, genetic screening, etc.) impossible. Management of PDIS during HUS-TTP is challenging because of the rapidly extensive course of distal necrosis [2]. Also, initiation of anticoagulation, especially heparin, should be prudent, mainly because of the underlying thrombocytopenia and acute renal failure $[7,8]$. Unfortunately, our two cases had a bad prognosis because of the rapidly extensive ischemia and poor neurological and renal outcomes.

\section{Conclusion}

Acral necrosis during MA is rare. A few pediatric cases were reported. Its prevalence increases after surgical and vascular interventions in genetically predisposed individuals. It is more frequent in the course of TTP than HUS. Management is challenging, and distal amputation is often necessary.

\section{Consent}

Patients' parents' consent to publication has been obtained.

\section{Conflicts of Interest}

The authors declare that they have no Conflicts of Interest.

\section{References}

[1] M. Batra, P. Tandon, and N. Gupta, "Clinical approach to a patient with isolated digital ischaemia," Journal of Indian Academy of Clinical Medicine, vol. 3, no. 1, pp. 23-28, 2002.

[2] J. C. Chang, "TTP-like syndrome: novel concept and molecular pathogenesis of endotheliopathy-associated vascular microthrombotic disease," Thrombosis Journal, vol. 16, no. 1, p. 20, 2018.

[3] N. Agarwal and B. Cherascu, "Concomitant acral necrosis and haemolytic uraemic syndrome following ingestion of quinine," Journal of Postgraduate Medicine, vol. 48, no. 3, p. 197, 2002.

[4] C. Bibbo and W. H. Davis, "Foot manifestations of the thrombotic thrombocytopenic purpura hemolytic-uremic syndrome: a review and case report," The Journal of Foot and Ankle Surgery, vol. 44, no. 4, pp. 301-306, 2005.

[5] J. C. Chang and S. K. Kathula, "Various clinical manifestations in patients with thrombotic microangiopathy," Journal of Investigative Medicine, vol. 50, no. 3, pp. 201-206, 2002.

[6] J. C. Chang and N. Ikhlaque, "Peripheral digit ischemic syndrome can Be a manifestation of postoperative thrombotic thrombocytopenic purpura," Therapeutic Apheresis and Dialysis, vol. 8, no. 5, pp. 413-418, 2004.

[7] J. Hofer, A. Rosales, C. Fischer, and T. Giner, "Extra-renal manifestations of complement-mediated thrombotic microangiopathies," Frontiers in Pediatrics, vol. 2, 2014.

[8] D. J. Saltzman, J. C. Chang, J. C. Jimenez et al., "Postoperative thrombotic thrombocytopenic purpura after open heart operations," The Annals of Thoracic Surgery, vol. 89, no. 1, pp. 119-123, 2010. 